\title{
Optimality Conditions for a Class of Optimization Problem in Banach Spaces
}

\author{
Xuanwei Zhou* \\ School of Basic Courses, Zhejiang Shuren University, Hangzhou 310015, China \\ ${ }^{*}$ Corresponding author
}

\begin{abstract}
In this paper, a class of optimization problem is studied. The objective function is a functional in a Banach space and the constraint is cone constraint where the cone doesn't need nonempty interior. The concept of the conjugate function is introduced and a duality theorem is established. Then, by use of the duality theorem and Robinson's constraint qualification, some optimality conditions for the optimization problems in Banach space are obtained.
\end{abstract}

Keywords—banach space; duality theory; optimality condition; optimization problem

\section{INTRODUCTION}

Optimality condition is an important topic in optimization: it is useful not only for identifying solutions of the problem, but it is also crucial in designing numerical methods for approximating the solutions. It is well known that duality theorem is very useful for optimality condition in optimization problem (see [1]-[10]).

In 1961 Wolfe first obtained a duality theorem for differentiable convex programming(see [11]). Afterwards, a number of different duality theorems distinct from the Wolfe dual are proposed for the nonlinear programs by Mond and so on (see [12]). Duality theorems for multiobjective programming problems with generalized convexity conditions were given by several authors (see [13]-[18]) . In these papwrs, by using the duality theorems, optimality conditions of optimization problems are also obtained in finite dimensional space.

In this paper, we give some optimality conditions of optimization problem in infinite dimensional space. The objective function is a functional of Banach space and the constraint is cone constraint. Specially, the constraint cone don't need nonempty interior. The concept of the conjugate function is introduced and a duality theorem is established. Then, by use of the duality theorem and Robinson's constraint qualification, the optimality condition for the optimization problems is obtained.

\section{DEFINITIONS AND LEMMAS}

Let $X$ be a Banach space, let $X *$ be the dual of $X$. A subset $K$ of $X$ is said to be a convex cone if

$$
\alpha x_{1}+\beta x_{2} \in K, \forall x_{1}, x_{2} \in K, \forall \alpha, \beta \geq 0
$$

A positive cone is a convex cone with apex at the origin.

A Banach space $X$ with a convex cone is said to be an ordered Banach space. The partial order on $X$ is defined by $x_{1} \leq_{K} x_{2}$ if and only if $x_{2}-x_{1} \in K, x_{1} \leq_{\text {int } K} x_{2}$ if and only if $x_{2}-x_{1} \in \operatorname{int} K$ if int $K \neq \varnothing$, where int $K$ is the interior of $K$.

Setting $K^{*}=\left\{\xi \in X^{*}:\langle\xi, x\rangle \geq 0, \forall x \in K\right\}, K^{*}$ is said to be the dual cone of the positive cone $K$.

Definition 1 Let $f: X \rightarrow R \cup\{ \pm \infty\}$ be a real valued function. It is said that $f$ is proper if its domain is nonempty and $f(x)>-\infty(\forall x \in X)$. It is said that $f$ is convex if $\forall x_{1}, x_{2} \in \operatorname{dom}(f)$, and $\forall t \in(0,1)$,

$$
t f\left(x_{1}\right)+(1-t) f\left(x_{2}\right) \geq f\left(t x_{1}+(1-t) x_{2}\right)
$$

Definition 2. Let $X$ be a Banach space, let $Y$ be an ordered Banach space with a positive cone $K$. A function $G: X \rightarrow Y$ is said to be $K$-convex on $X$ if and only if $\forall x_{1}, x_{2} \in X, \forall t \in(0,1)$,

$$
t G\left(x_{1}\right)+(1-t) G\left(x_{2}\right)-G\left(t x_{1}+(1-t) x_{2}\right) \in K
$$

Definition3. Let $X$ be a Banach space, let $X *$ be the dual of $X$, and let $f: X \rightarrow R \cup\{ \pm \infty\}$ be a real valued (possibly nonconvex) function. A function $f^{*}: X^{*} \rightarrow$ $R \cup\{ \pm \infty\}$ defined by

$$
f^{*}\left(x^{*}\right)=\sup _{x \in X}\left\{\left\langle x^{*}, x\right\rangle-f(x)\right\}
$$


is called the conjugate function of $f$. In a similar way, given $g: X^{*} \rightarrow R \cup\{ \pm \infty\}$, its conjugate $g^{*}: X \rightarrow R \cup\{ \pm \infty\}$ is defined as

$$
g *(x)=\sup _{x^{*} \in X^{*}}\left\{\left\langle x^{*}, x\right\rangle-g\left(x^{*}\right)\right\}
$$

Then

$$
f^{* *}(x)=\sup _{x^{*} \in X^{*}}\left\{\left\langle x^{*}, x\right\rangle-f *\left(x^{*}\right)\right\}
$$

The following results are well known.

Lemma1 (Fenchel-Moreau-Rockafellar) Let $f: X \rightarrow$ $R \cup\{ \pm \infty\}$ be a real valued function. Then

$$
f^{* *}=\operatorname{cl}(\operatorname{convf}) .
$$

The above lemma implies that $f^{* *}=f$ if and only if $f$ is convex and closed.

Lemma2 Let $f: X \rightarrow R \cup\{ \pm \infty\}$ be a real valued convex function. If $f$ is continuous at 0 , then $f=\operatorname{cl}(f)$.

\section{OPtiMizATION PROBLEM}

In this section, we discuss the following optimization problem.

Let $X$ be a Banach space, let $Y$ be an ordered Banach space with a positive cone $K$. We consider the program

$$
\min f(x) \text {, s.t. } G(x) \in-K \text {, }
$$

where $f: X \rightarrow R, G: X \rightarrow Y, K \subset Y$ is a convex closed cone.

The above problem (P) has the feasible set

$$
D=\{x \in X: G(x) \in-K\} .
$$

Denote $I_{-K}(\cdot)$ the indicator function of a nonempty set $-K$, then problem $(\mathrm{P})$ takes the form

$$
\min _{x \in X}\left\{f(x)+I_{-K_{1}}(G(x))\right\}
$$

embed (P) into the family of optimization problems

$$
\left(P_{y}\right) \quad \min _{x \in X}\left\{f(x)+I_{-K_{1}}(G(x)+y)\right\},
$$

where $y \in Y$ is the parameter vector. Clearly, for $y=0$, the corresponding problem $\left(P_{0}\right)$ coincides with the problem (P). Problem $\left(P_{y}\right)$ amounts to minimization, with respect to $X$, of the function

$$
\varphi(x, y)=f(x)+I_{-K}(G(x)+y) .
$$

Denote the corresponding optimal value function by $v(y)$, that is $v(y)=\operatorname{val}\left(P_{y}\right)$,

or, equivalently, $v(y)=\inf _{x \in X} \varphi(x, y)$.

It is easy to know

$$
v^{* *}(y)=\sup _{y^{*} \in Y^{*}}\left\{\left\langle y^{*}, y\right\rangle-\varphi^{*}\left(0, y^{*}\right)\right\}
$$

This leads to the following definition of the dual problem:

$$
\left(D_{y}\right) \quad \max _{y^{*} \in Y^{*}}\left\{\left\langle y^{*}, y\right\rangle-\varphi^{*}\left(0, y^{*}\right)\right\},
$$

We refer to the above problem $\left(D_{y}\right)$ as the conjugate dual of $\left(P_{y}\right)$. In particular, for $y=0$ the corresponding problem ( $D_{0}$ ) becomes

$$
\max _{y^{*} \in Y^{*}}\left\{-\varphi^{*}\left(0, y^{*}\right)\right\},
$$

and is viewed as the dual of $(P)$. Clearly, $\operatorname{val}\left(P_{y}\right)=v(y)$ and $\operatorname{val}\left(D_{y}\right)=v^{* *}(y)$.

Lemma3 Let $K$ be a convex closed cone and $G: X \rightarrow Y$ be $K$-convex on $X$, then $I_{-K}(G(x)+y)$ is convex.

Proof. It is only to show that the set $\{(x, y) \in X \times Y: G(x)+y \in-K\}$ is convex. If for any $\left(x_{1}, y_{1}\right),\left(x_{2}, y_{2}\right) \in\{(x, y) \in X \times Y: G(x)+y \in-K\} \quad$, then

$$
G\left(x_{1}\right)+y_{1} \in-K, G\left(x_{2}\right)+y_{2} \in-K .
$$

Since $G$ is $K$-convex, for any $t \in[0,1]$, 


$$
t G\left(x_{1}\right)+(1-t) G\left(x_{2}\right)-G\left(t x_{1}+(1-t) x_{2}\right) \in K .
$$

So

$$
\begin{gathered}
G\left(t x_{1}+(1-t) x_{2}\right)+t y_{1}+(1-t) y_{2} \\
\in t G\left(x_{1}\right)+(1-t) G\left(x_{2}\right)-K+t y_{1}+(1-t) y_{2} \\
\in-t K-(1-t) K-K \subset-K .
\end{gathered}
$$

It means that $\{(x, y) \in X \times Y: G(x)+y \in-K\}$ is convex.

Lemma4 If the function $f(x)$ is convex, then the optimal value function $v(y)$ is convex.

Proof. Clearly, $\varphi(x, y)=f(x)+I_{-K}(G(x)+y)$ is convex, then for any $x_{1}, x_{2} \in X, y_{1}, y_{2} \in Y$ and $t \in[0,1]$,

$$
t \varphi\left(x_{1}, y_{1}\right)+(1-t) \varphi\left(x_{2}, y_{2}\right) \geq \varphi\left(t x_{1}+(1-t) x_{2}, t y_{1}+(1-t) y_{2}\right.
$$
$\geq v\left(t y_{1}+(1-t) y_{2}\right)$.

Minimizing the left hand side over $x_{1}$ and $x_{2}$, we obtain

$$
t v\left(y_{1}\right)+(1-t) v\left(y_{2}\right) \geq v\left(t y_{1}+(1-t) y_{2}\right),
$$

which shows that $v(y)$ is convex.

Lemma5 Suppose that (i) the function $f: X \rightarrow R$ is convex and continuous, and $K$ is a convex closed cone, and $G: X \rightarrow Y$ is $K$-convex and continuous; (ii) $\operatorname{val}(P)$ is finite; (iii) the set $S(D)$, of optimal solutions of the dual problem, is nonempty; (iv) the set $G(X)+K$ has a nonempty relative interior. Then $\operatorname{val}(P)=\operatorname{val}(D)$

Proof. Since $\operatorname{val}(D)=v^{* *}(0)$ and $S(D)$ is nonempty, we have that $v^{* *}(0)$ is finite. By that $S(D)=\partial v^{* *}(0)$, we have that $\partial v^{* *}(0)$ is nonempty. This implies that $N_{\text {dom }\left(v^{* *}\right)}(0)=\{0\}$. Since $\operatorname{dom}\left(v^{* *}\right)$ coincides with the topological closure of $\operatorname{dom}(v)$, it follows that the linear space generated by $\operatorname{dom}(v)$ is dense in $Y$. Since it is assumed that $\operatorname{dom}(v)$ has a nonempty relative interior, we have then that the interior of $\operatorname{dom}(v)$ is nonempty. Consequently, by the convex sets separation theorem, 0 can be separated from $\operatorname{dom}(v)$ if and only if $0 \notin \operatorname{int}(\operatorname{dom}(v))$. Clearly, if 0 can be separated from $\operatorname{dom}(v)$, then $N_{\text {dom }(v)}(0)=N_{\text {dom }\left(v^{* *}\right)}(0) \neq\{0\}$. Therefore, we obtain that $0 \in \operatorname{int}(\operatorname{dom}(v))$. It follows that $v(y)$ is continuous at 0 , and hence by lemma1 and lemma2, $\operatorname{val}(P)=\operatorname{val}(D)$.

Theorem1 Suppose that (i) the function $f: X \rightarrow R$ is convex and continuous, and $K$ is a convex closed cone, and $G: X \rightarrow Y$ is $K$-convex and continuous; (ii) $\operatorname{val}(P)$ is finite; (iii) the set $S(D)$, of optimal solutions of the dual problem, is nonempty; (iv) the set $G(X)+K$ has a nonempty relative interior. If $x_{0} \in X$ is optimal solution of problem (P), then there exists $\bar{y}^{*} \in K^{+}$such that

$$
x_{0} \in \underset{x \in X}{\arg \min } L\left(x, \bar{y}^{*}\right) \text { and }\left\langle\bar{y}^{*}, G\left(x_{0}\right)\right\rangle=0,
$$

where $L\left(x, y^{*}\right)=f(x)+\left\langle y^{*}, G(x)\right\rangle$ is the Lagrangian function of the problem $(\mathrm{P})$.

$y_{2}$ Proof. Note that the domain of the function $\varphi(x, y)=f(x)+I_{-K}(G(x)+y)$ is nonempty. Indeed, since $f$ and $I_{-K}$ are proper, we take $y \in-K$. Then

$$
\varphi(x, y-G(x))=f(x)+I_{-K}(y)=f(x)<+\infty,
$$

and hence $(x, y-G(x)) \in \operatorname{dom}(\varphi)$. Moreover, $\varphi(x, y)>-\infty(\forall(x, y) \in X \times Y)$, and hence $\varphi(x, y)$ is proper. The function $\varphi(x, y)$ is lower semicontinuous since $I_{-K}$ is lower semicontinuous, and $f$ and $G$ are continuous.

The conjugate function of $\varphi(x, y)$,

$$
\begin{aligned}
& \varphi^{*}\left(x^{*}, y^{*}\right) \\
& =\sup _{x \in X, y \in Y}\left\{\left\langle x^{*}, x\right\rangle+\left\langle y^{*}, y\right\rangle-f(x)-I_{-K}(G(x)+y)\right\} \\
& =\sup _{x \in X}\left\{\left\langle x^{*}, x\right\rangle-f(x)-\left\langle y^{*}, G(x)\right\rangle\right\} \\
& +\sup _{y \in Y}\left\{\left\langle y^{*}, G(x)+y\right\rangle-I_{-K}(G(x)+y)\right\} \\
& =\sup _{x \in X}\left\{\left\langle x^{*}, x\right\rangle-f(x)-\left\langle y^{*}, G(x)\right\rangle\right\} \\
& +\sup _{y \in Y}\left\{\left\langle y^{*}, G(x)+y\right\rangle-I_{-K}(G(x)+y)\right\} \\
& =\sup _{x \in X}\left\{\left\langle x^{*}, x\right\rangle-L\left(x, y^{*}\right)\right\}+\sup _{y \in Y}\left\{\left\langle y^{*}, y\right\rangle-I_{-K}(y)\right\} \\
& =\sup _{x \in X}\left\{\left\langle x^{*}, x\right\rangle-L\left(x, y^{*}\right)\right\}+I_{-K} *\left(y^{*}\right)
\end{aligned}
$$

The dual problem $\left(D_{y}\right)$ can be written in the form 
$\left.\left(D_{y}\right) \quad \max _{y^{*} \in Y^{*}}\left\{\left\langle y^{*}, y\right\rangle-\inf _{x \in X} L\left(x, y^{*}\right)\right\}-I_{-K}^{*}\left(y^{*}\right)\right\}$

In particular, for $y=0$, the dual of $(\mathrm{P})$ is

$$
\max _{y^{*} \in Y^{*}}\left\{\left\langle y^{*}, y\right\rangle+\inf _{x \in X} L\left(x, y^{*}\right)-I_{-K} *\left(y^{*}\right)\right\}
$$

From lemma5, $\operatorname{val}(P)=\operatorname{val}(D)$, and there exist the optimal solution $x_{0} \in X$ of problem $(\mathrm{P})$ and the optimal solution $\bar{y}^{*} \in Y^{*}$ of dual problem $(D)$ such that

$$
f\left(x_{0}\right)+I_{-K}\left(G\left(x_{0}\right)\right)=\inf _{x \in X} L\left(x, \bar{y}^{*}\right)-I_{-K}^{*}\left(\bar{y}^{*}\right)
$$

This is

$$
\begin{aligned}
& \left(L\left(x_{0}, \bar{y}^{*}\right)-\inf _{x \in X} L\left(x, \bar{y}^{*}\right)\right)+\left(I_{-K}\left(G\left(x_{0}\right)\right)\right. \\
& \left.+I_{-K} *\left(\bar{y}^{*}\right)-\left\langle\bar{y}^{*}, G\left(x_{0}\right)\right\rangle\right)=0 .
\end{aligned}
$$

Clearly, $L\left(x_{0}, \bar{y}^{*}\right)-\inf _{x \in X} L\left(x, \bar{y}^{*}\right) \geq 0$ and

$$
I_{-K}\left(G\left(x_{0}\right)\right)+I_{-K} *\left(\bar{y}^{*}\right)-\left\langle\bar{y}^{*}, G\left(x_{0}\right)\right\rangle \geq 0 .
$$

Therefore, $L\left(x_{0}, \bar{y}^{*}\right)-\inf _{x \in X} L\left(x, \bar{y}^{*}\right)=0$,

$$
I_{-K}\left(G\left(x_{0}\right)\right)+I_{-K} *\left(\bar{y}^{*}\right)-\left\langle\bar{y}^{*}, G\left(x_{0}\right)\right\rangle=0 .
$$

The equality $L\left(x_{0}, \bar{y}^{*}\right)-\inf _{x \in X} L\left(x, \bar{y}^{*}\right)=0$ means $x_{0} \in \arg \min L\left(x, \bar{y}^{*}\right) \quad$, and another equality $I_{-K}\left(G\left(x_{0}\right)\right)+I_{-K} *\left(\bar{y}^{*}\right)-\left\langle\bar{y}^{*}, G\left(x_{0}\right)\right\rangle=0$ holds if and only if $\bar{y}^{*} \in \partial I_{-K}\left(G\left(x_{0}\right)\right)=N_{-K}\left(G\left(x_{0}\right)\right)$. Since $K$ is a convex cone, the condition $\bar{y}^{*} \in N_{-K}\left(G\left(x_{0}\right)\right)$ is equivalent to $\bar{y}^{*} \in K^{+}$and $\left\langle\bar{y}^{*}, G\left(x_{0}\right)\right\rangle=0$.

Now we consider the smooth (not necessarily convex) case.

Theorem2 Suppose that the function $f: X \rightarrow R$ and the mapping $G: X \rightarrow Y$ are continuously differentiable. If $x_{0} \in X$ is optimal solution of problem (P), and

the Robinson's constraint qualification

$$
0 \in \operatorname{int}\left\{G\left(x_{0}\right)+D G\left(x_{0}\right)(X)+K\right\}
$$

holds, then there exists $\bar{y}^{*} \in K^{+}$such that $D L\left(x_{0}, \bar{y}^{*}\right)=0$ and $\left\langle\bar{y}^{*}, G\left(x_{0}\right)\right\rangle=0$.
Proof. We will show that $h=0$ is an optimal solution of the linearize problem

$$
\left(P_{L}\right) \quad \min _{x \in X} D f\left(x_{0}\right) h \text {, s.t. } G\left(x_{0}\right)+D G\left(x_{0}\right) h \in-K .
$$

Indeed, if $h \in\left\{h \in X: G\left(x_{0}\right)+D G\left(x_{0}\right) h \in-K\right\}$, then by the Robinson's constraint qualification, there exist sequences $t_{n} \rightarrow 0^{+}$and $x_{n}=x_{0}+t_{n} h+o\left(t_{n}\right)$ such that $x_{n} \in D$. Since $x_{0}$ is optimal solution of problem (P), it follows that

$$
D f\left(x_{0}\right) h=\lim _{n \rightarrow \infty} \frac{f\left(x_{n}\right)-f\left(x_{0}\right)}{t_{n}} \geq 0
$$

and consequently $h=0$ is an optimal solution of the problem $\left(P_{L}\right)$. Replacing $f(x)$ with $D f\left(x_{0}\right) x$ and $G(x)$ with $G\left(x_{0}\right)+D G\left(x_{0}\right) x$ in theorem1, we have that there exists $\bar{y}^{*} \in K^{+}$such that $D L\left(x_{0}, \bar{y}^{*}\right)=0$ and $\left\langle\bar{y}^{*}, G\left(x_{0}\right)\right\rangle=0$.

\section{CONCLUSION}

Optimality condition for optimization problems is a very active field. Among important contributions that were not quoted yet (see[19]-[22]), we only mention the work based on constraint qualification and duality theorem.

This paper presents a new type of optimization problems in infinite dimensional Banach space. This new optimization problem extend finite dimensional space to infinite dimensional and inequality constraints to cone constraint. The constraint cone doesn't need nonempty interior that generalizes the classical one, when the interior of constraint cone is nonempty. A duality theorem is established by using Robinson's constraint qualification. Then the optimality condition for this optimization problem is obtained.

As pointed out by an anonymous referee, we will study then how to obtain first order sufficient optimality conditions and general second optimality conditions for this optimization problem, and how to discuss composite optimization, the case of nonisolated optimal solutions and specific aspects such as quadratic programming, reduction procedures, and exact penalty functions.

\section{REFERENCES}

[1] A. Auslender, "Existence of optimal solutions and duality results under weak conditions. ” Math. Program.Ser. A, vol. 88, pp45-59, 2000.

[2] J. Gwinner and W. Oettli, "Theorems of the Alternative and Duality for Inf-Sup Problems," Mathematics of Operations Research, Vol. 19, pp. 238-256, 1994.

[3] D. T. Luc , “ On Duality Theorems in. Multiobjective Programming,” Journal of Optimization Theory and Applications, Vol. 48, pp. 557-582, 1984. 
[4] V. Michel, "Theorems of the Alternative for Multivalued Mappings and Applications to Mixed ConvexlConcave Systems of Inequalities,” SetValued Analysis, Vol. 18, pp. 601-616, 2010.

[5] C. Singh and M. A. Hanson, "Saddle-Point Theory for Nondifferentiable Programming," Journal of Information and Optimization Science, Vol. 7 , pp. $41-48,1986$.

[6] Z. A. Liang, H. X.Huang and P. M. Pardalos, "Optimality conditions and duality for a class of nonlinear fractional programming problems. ” J. Optim. Theory Appl. Vol.110, pp. 611-619,2001.

[7] R. I. Bot, E. R. Csetnek and A. Moldovan, "Revisiting some duality theorems via the quasirelative interior in convex optimization. ” J. Optim. Theory Appl. Vol.139, pp.67-84 (2008)

[8] V. Jeyakumar, "Constraint qualifications characterizing Lagrangian duality in convex optimization. " J. Optim. Theory Appl. Vol.136, pp.31-41, 2008.

[9] V. Jeyakumar and G. M. Lee, "Complete characterizations of stable Farkas' lemma and cone-convex programming duality. ” Math. Prog. Vol. A 114, pp.335-347, 2008.

[10] V. Jeyakumar and G. Y. Li, "Stable zero duality gaps in convex programming: complete dual characterizations with applications to semidefinite programs.” J. Math. Anal. Appl. Vol.360, pp.156-167, 2009.

[11] P. Wolfe, “A duality theorem for non-linear programming,” Quarterly of Applied Mathematics, vol. 19, pp. 239-244, 1961.

[12] B. Mond and T. Weir, "Generalized concavity and duality," in Generalized Concavity in Optimization and Economics, S. Schaible and W. T. Ziemba, Eds., pp. 263-279, Academic Press, New York, NY, USA, 1981.

[13] E. Ning, W.Song and Y. Zhang, "Second order sufficient optimality conditions in vector optimization," Journal of Global Optimization, Vol.54, pp.537-549, 2012.

[14] R. R. Egudo and M. A. Hanson, "On sufficiency of Kuhn-Tucker conditions in nonsmooth multiobjective programming," Technical Report M-888, Florida State University, Tallahassee, Fla, USA, 1993.

[15] D. S. Kim and S. Schaible, "Optimality and duality for invex nonsmooth multiobjective programming problems," Optimization, vol. 53, no. 2, pp. 165-176, 2004.

[16] S. K. Mishra and R. N. Mukherjee, "On generalised convex multiobjective nonsmooth programming," Australian Mathematical Society, vol. 38, no. 1, pp. 140-148, 1996

[17] B. Mond and M. Schechter, "Nondifferentiable symmetric duality," Bulletin of the Australian Mathematical Society, vol. 53, no. 2, pp. 177188, 1996.

[18] S. Nobakhtian, "Optimality and duality for nonsmooth multiobjective fractional programming with mixed constraints,” J. Glob. Optim. vol.41, pp.103-115, 2008.

[19] K. Ramanathan and K.C.Sivakumar, "Theorems of the Alternative over Indefinite Inner Product Spaces,” Journal of Optimization Theory and Applications, Vol. 137, pp. 99-104,2008.

[20] X. M. Yang , X. Q. Yang and G. Y. Chen, "Theorems of the Alternative and Optimization," Journal of Optimization Theory and Applications, Vol. 107, pp. 627-640,2000.

[21] R. ZENG, "Generalized Gordon Alternative Theorem with Weakened Convexity and Its Applications,”Optimization, Vol. 51, pp. 709-717, 2002.

[22] S. Paeck, "Convexlike and Concavelike Conditions in Alternative, Minimax, and MinimizationTheorems," Journal of OptimizationTheory and Applications,Vol.74, pp.317-332, 1992. 